\title{
The diagnostic value of MR spectroscopy versus DWI-MRI in therapeutic planning of suspicious multi-centric cerebral lesions
}

\author{
Hoda Abdel Kareem Ahmed ${ }^{1 *}$ and Hoda Mokhtar ${ }^{2}$
}

\begin{abstract}
Background: A broad spectrum of non-neoplastic lesions can radiologically mimic cerebral neoplasms. Magnetic resonance imaging $(\mathrm{MRI})$, magnetic resonance spectroscopy (MRS), and diffusion-weighted imaging (DWI) are the most extensively used for enabling lesional characterization of different brain disorders. We aimed to assess the diagnostic value of MRS versus DWI in the diagnosis and therapeutic planning of multicentric cerebral focal lesions and in our retrospective study, we enrolled 64 patients with 100 brain lesions who underwent pre- and postcontrast MRI, MRS, and DWI. Diagnoses supplied by the histopathology and follow up clinical results as a gold standard. Sensitivity, specificity, positive predictive value (PPV), negative predictive value (NPV), and diagnostic accuracy were calculated.

Results: Conventional MRI poorly differentiates multiple cerebral lesions with $89.33 \%$ sensitivity, $44.4 \%$ specificity, and $78 \%$ accuracy. MRS results revealed statistical significance for differentiating neoplastic from non-neoplastic lesions as regards $\mathrm{Cho} / \mathrm{Cr}$, Cho/NAA, and NAA/Cr ratios $(\mathrm{M} \pm \mathrm{SD}$ ) with $P<0.001$ (significant), and there is statistical significance for neoplastic lesion differentiation when Cho/NAA and $\mathrm{Ch} / \mathrm{Cr}$ ratios measured in the pre-lesional areas outside the tumor margin. DWI showed mixed diffusion changes in most of the studied lesions and the measured $A D C$ values ranges showed overlap in neoplastic and non-neoplastic lesions, $P$ value $=0.236^{*}$ (insignificant).
\end{abstract}

Conclusion: MRS was found to be a more accurate diagnostic tool than DWI with ADC measurements in the differentiation and therapeutic planning of multicentric cerebral focal lesions.

\section{Background}

A broad spectrum of non-neoplastic focal lesions can radiologically mimic cerebral neoplasms that offer a diagnostic challenge to both the clinician and radiologist [1]. Accurate, non-invasive diagnosis is important, as the prognosis and therapeutic plan are often based on the histopathological grade of the tumor [2, 3]. MRI provides an initial diagnosis of intracranial mass lesions [4]. MR spectroscopy (1H-MRS) is a diagnostic non-invasive imaging that offers the possibility of detecting and quantifying different metabolites of the brain; MRS has

\footnotetext{
*Correspondence: Hodaradiology2012@gmail.com

${ }^{1}$ Radio-diagnosis Department, Faculty of Medicine, Al-Azhar University, Assuit, Egypt

Full list of author information is available at the end of the article
}

clinically been most extensively used for assessing brain disorders [5-7]. Inborn errors of metabolism can lead to accumulation of metabolites and consequently abnormal peaks in 1H-MRS. Assignment of the resonance peaks of $\mathrm{N}$-acetyl-aspartate (NAA) at $2.02 \mathrm{ppm}$, creatinine $(\mathrm{Cr})$ at $3.03 \mathrm{ppm}$ and $3.9 \mathrm{ppm}$, choline $(\mathrm{Ch})$ at $3.2 \mathrm{ppm}$, and lipid at $1-1.6 \mathrm{ppm}$, as well as the metabolite ratios of NAA/ $\mathrm{Cr}$ and $\mathrm{Ch} / \mathrm{Cr}$, should be calculated [8]. By using standardized post-processing protocols, the raw data processed automatically, allowing for operator-independent quantifications [9]. MRS and other physiological imaging techniques may assist the surgeon in planning targeted radiotherapy, as well as to help to differentiate residual or recurrent tumor from radiation necrosis on follow-up [10]. Diffusion-weighted images (DWI) have been 
Table 1 Histo-pathological results and final clinical diagnosis of the studied 100 lesions

\begin{tabular}{ll}
\hline Lesions number & Final diagnosis \\
\hline 37 & Metastasic deposits \\
16 & GBM \\
10 & Multiple sclerosis \\
7 & Post irradiation lesions \\
6 & Lymphoma \\
7 & Infarctions \\
6 & Anaplastic astrocytoma \\
4 & Brain abscesses \\
3 & Tuberculoma \\
2 & Focal encephalitis \\
2 & Vacultitis \\
100 & Total \\
\hline
\end{tabular}

increasingly used to characterize solid masses aiding in the distinction between benign and malignant lesions [11]. DW-MRI detects Brownian motion of water protons; thus, reflecting the biological character of tissue and the apparent diffusion coefficient (ADC) is used to quantify this Brownian motion [12]. The signal intensity reduction on DW-MRI can be quantified by calculating the ADC values which can be calculated from a series of DW-MRI at different $b$ values [13]. DWI and the calculated ADC values from the core of the lesion added more information to MRI in the differentiation and grading of brain tumors $[14,15]$.

The purpose of this study is to assess the diagnostic value of MRS versus DWI in the diagnosis and therapeutic planning of multicentric suspicious cerebral focal lesions.

\section{Methods}

\section{Patient's demographic data}

This prospective study included 64 patients with 100 brain focal lesions, 39 males and 25 females, their ages ranged from $11-78$ years with mean age \pm 43 Ys who referred to MRI unite with a diagnosis of multiple suspicious brain focal lesions. An informed consent was obtained from all patients. Patients who had general contraindications for MRI examination or noisy/non-diagnostic MRS or DWI examinations due to motion artifacts were excluded from this study.

\section{MRI protocol and technique}

All MRI examinations were obtained using 1.5 tesla superconducting unit in the form of conventional sequences of routine brain, including the following.

\section{Conventional MRI}

Pre-contrast axial and sagittal T1 and contrast enhanced axial, sagittal, and coronal T1W spin-echo images after IV administration of Gad-DTPA "dose, $0.1 \mathrm{mmol} / \mathrm{kg}$ " (TR/TE, 600/11); axial FLAIR (10 000/120; inversion time msec, 2200); coronal T2W fast spin-echo (TR/TE, 3000-4000/98); Images FOV: $220 \mathrm{~mm}$ with $5 \mathrm{~mm}$ slice thickness.

\section{DW-MRI prior to contrast-enhanced examination}

This was performed with a two-dimensional echo-planar imaging (EPI) sequence (TR/TE, 4045/127 ms, matrix $152 \times 105,5 \mathrm{~mm}$ thickness with $1 \mathrm{~mm}$ slice gap, and $124 \mathrm{~s}$ duration) in the axial plane. The ADC map images were created automatically by the system after taking three different $b$ values $\left(b=0,500\right.$ and $\left.1000 \mathrm{~s} / \mathrm{mm}^{2}\right)$. Mean ADC value measured by placing ROI of about 1 $\mathrm{ccm}$ in the solid part of the lesion as well as in the prelesional regions and expressed in $10^{-3} \mathrm{~mm}^{2} / \mathrm{s}$.

\section{MR-Spectroscopy}

Multivoxel MRS was done and water suppression was achieved with a chemical shift selection technique. Max. high Cho/NAA, Cho/Cr, Cho/NAA + $\mathrm{Cr}$ at intermediate $\mathrm{TE} 144 \mathrm{msec}$ and $\mathrm{ml} / \mathrm{Cr}$ at short TE $35 \mathrm{msec}$ peak ratios in the lesions and in the perilesional areas away from

Table 2 Conventional MRI findings of the all studied lesions

\begin{tabular}{|c|c|c|c|c|c|c|}
\hline No & Texture (uniform/heterogen.) & Edema degree & Mass effect & Enhancing pattern & MRI diagnosis & Final diagnosis \\
\hline 37 & $9 / 28$ & IIIIII & $+++v e$ & 15 ring, 19 nodular & 29 Metast. 5 HGG & Metast. \\
\hline 16 & 16 heterogen. & II-III & $+++v e$ & 16 heterogen. & 11 HGG, 5 metastatic & GBM \\
\hline 10 & $6 / 4$ & I-II & $++v e$ & 6 non $/ 2$ ring, 2 nodular & 9 MS, 1 neoplasm & MS \\
\hline 7 & 7 heterogen. & $I-I I$ & $++v e$ & 5 hetrogen. 2 nodular & 6 Post-irrad, 1 Recur & Post-rad. \\
\hline 6 & $5 / 1$ & I & $+\mathrm{ve}$ & 4 ring, 2 non & 4 Lymph. 2 metast & Lymph. \\
\hline 7 & 7 & $I-I I$ & $++v e$ & 6 non, 1 hetrog. & 5 infarcts 2 ?neoplasm & Infarcts \\
\hline 6 & 6 heterogen. & II-III & $+++v e$ & 2 ring/5 heterogen. & Neoplastic?hgg/?metas & A.astrocyt \\
\hline 4 & Non-homogonous & $I-I I$ & $++v e$ & 2 ring/nodul. & 2 abscess, 2 neoplastic & Abscess \\
\hline 3 & Non-homogonous & I & $+\mathrm{ve}$ & 3 ring & 3 T.B. & T.B. \\
\hline 2 & Non-homogonous & I-II & $++v e$ & 2 patchy & 2 Enceph. & Encephal. \\
\hline 2 & Non-homogonous & I-II & $++v e$ & 2 patchy & ?Inflamm ?neoplastic & Vacultitis \\
\hline
\end{tabular}


Table 3 Spectroscopic data analysis for all non-neoplastic and neoplastic lesions

\begin{tabular}{llll}
\hline MRS $($ mean \pm SD) & Neoplastic No $=65$ & Non-neoplastic No $=35$ & $P$ value \\
\hline Cho/Cr & $5.97 \pm 6.01$ & $1.87 \pm 0.5$ & $<0.001^{*}$ \\
Cho/NAA & $6.22 \pm 5.07$ & $1.3 \pm 0.69$ & $<0.001^{*}$ \\
NAA/Cr & $0.81 \pm 0.71$ & $1.29 \pm 0.69$ & $0.003^{*}$ \\
Cho/NAA + Cr & $2.81 \pm 2.38$ & $0.7 \pm 0.4$ & $<0.001^{*}$ \\
NAA/Cho & $0.39 \pm 0.3$ & $1.4 \pm 0.5$ & $<0.001^{*}$ \\
Lipid & $44+$ ve, 21-ve & $25+$ ve, 10-ve & - \\
Lactate & $51+$ ve, 14-ve & $25+$ ve, 10-ve & - \\
(AA, A, S, and P) & 65 -ve & + ve 3 abs/-ve 32 & - \\
\hline
\end{tabular}

CSF and scalp fat were recorded. Lipid signal presence or absence was recorded. 2D MRSI PRESS at intermediate TE $144 \mathrm{msec}$, voxel size inside spectroscopic grid 10 $\times 10 \mathrm{~mm}$, and the spectroscopic grid is extended and manually adjusted to include lesion, edema if present, and normal brain tissue ( $5 \mathrm{~min}$ ). 2D short TE sequence at $35 \mathrm{msec}$, voxel size inside spectroscopic grid $10 \times 10$ $\mathrm{mm}$, and the spectroscopic grid is extended and manually adjusted to include lesion, edema if present, and normal brain tissue (12 min).

Single voxel spectroscopy (SVS) short TE $35 \mathrm{msec}$ in selected cases $(n=18)$ as small size lesions near skull base or high vertex or in non-hemorrhagic area within hemorrhagic SOL (4 min). The peak ratios can be obtained by measuring and comparing areas under the curve (AUC). The peaks are assigned as follows (Cho peak at $3.2 \mathrm{ppm}, \mathrm{Cr}$ at $3 \mathrm{ppm}$, NAA peak at $2.02 \mathrm{ppm}$, mobile lipids at $0.5-1.5 \mathrm{ppm}$, lactate $1.44 \mathrm{ppm}$, and $\mathrm{mI}$ at $3.56 \mathrm{ppm})$.

\section{Image interpretation}

All MR images were evaluated by a neuro-imaging radiologist who was blinded to clinical data and only concentrated on conventional MRI signal changes, evaluation of DWI with ADC values calculations and analysis of the MRS peaks \& ratios from the all studied lesions and from areas outside lesions margins at the edema if any.

\section{Biopsy with the histopathology results or clinical diagnosis}

This was done after medical treatment and follow-up were taken as gold standard.

\section{Statistical analysis}

This was done to evaluate the utility of MRS and DWI with ADC value measurements in the diagnosis of multiple brain focal lesions. Data entry and analysis were done by using the program Statistical Package for the Social Sciences (SPSS). Both technique results were compared using Student's $t$ test, and sensitivity, specificity, negative, and positive predictive values (PPV) were assessed and compared. $P<0.05$ was considered statistically significant.

\section{Results}

This study was carried on 64 patients with 100 brain focal lesions that proved to be 37 metastatic deposits in 17 patients with known diagnosis of primary neoplasm, (16 lesions from breast cancer, 14 from lung cancer, 4 from prostatic cancer, and 3 lesions from renal cell Ca), 16 glioblastoma multiform (GBM), 10 multiple sclerosis, 7 post irradiation lesions, 6 lymphoma, 7 infarctions, 6 anaplastic astrocytoma, 4 brain abscesses, 3 tuberculoma, 2 focal encephalitis, and 2 vacultitis according to the histo-pathological results and final clinical diagnosis after medical treatment and follow-up (Table 1).

The conventional MRI (T1,T2, FLAIR, and the contrast enhanced T1WI) findings as regard the lesion texture, associated edema, mass effect, and the enhancement pattern suggest the correct diagnosis in $81 \%$ of lesions when correlated with histopathology and final clinical diagnosis. In Table 2, conventional MRI poorly differentiates multiple cerebral lesions as regards primary cerebral neoplasm or metastatic lesions; also there is overlap in diagnosis of some neoplastic and non-neoplastic lesion diagnosis by conventional MRI images with $89.33 \%$ sensitivity, $44.4 \%$ specificity, and $78 \%$ accuracy.

Table 4 Spectroscopic data intra-lesional within and outside tumor margin of the primary and metastatic neoplastic lesions

\begin{tabular}{llll}
\hline MRS $($ mean \pm SD) & Primary & Metastatic & $P$ value \\
\hline Cho/Cr & $7.21 \pm 4.36$ & $5.08 \pm 3.75$ & 0.071 \\
Cho/NAA & $9.31 \pm 6.41$ & $6.32 \pm 5.82$ & 0.089 \\
NAA/Cr & $0.57 \pm 0.5$ & $0.91 \pm 0.82$ & 0.167 \\
Cho/NAA + Cr & $3.21 \pm 2.32$ & $2.8 \pm 3.22$ & 0.181 \\
NAA/Cho & $0.2 \pm 0.2$ & $0.5 \pm 0.5$ & 0.165 \\
Cho/NAA & $323 \pm 2.42$ & $0.71 \pm 0.23$ & $<0.001^{*}$ \\
Cho/Cr & $2.53 \pm 1.38$ & $1.06 \pm 0.33$ & $<0.001^{*}$ \\
\hline
\end{tabular}


Table 5 DWI and ADC values for all neoplastic and non-neoplastic lesions

\begin{tabular}{|c|c|c|c|}
\hline$\overline{D W l}$ & Neoplastic (65) & Non-neoplastic (35) & $P$ value \\
\hline Restricted Free/mixed & Mixed/free 59/6 & Mixed/restricted-free, $4 / 22-9$ & - \\
\hline $\mathrm{ADC}$ range $(\mathrm{M} \pm \mathrm{SD})$ & $0.6-1.7,0.88 \pm 0.31$ & $0.37-3.9,0.91 \pm 0.61$ & $0.236 *$ insignificant \\
\hline
\end{tabular}

\section{MRS diagnosis}

According to the MRS results, we classified the studied 100 lesions into non-neoplastic and neoplastic lesions, then the neoplastic lesions further classified into primary and metastatic neoplastic lesions (Tables 3 and 4).

There was statistical significance for differentiation between neoplastic and non-neoplastic lesions by MRS as regards $\mathrm{Cho} / \mathrm{Cr}$, Cho/NAA, NAA/Cr, Cho/NAA $+\mathrm{Cr}$, and NAA/Cho ratios $(\mathrm{M} \pm \mathrm{SD})$ with $P<0.001$ (significant). Lipid peak was seen in 42 neoplastic lesions and in 27 of non-neoplastic, while lactate peaks seen in 49 neoplastic lesions and in 26 of non-neoplastic lesions and increased with tumor aggressiveness. Other metabolites such as amino acid (AA), acetate (A), and succinate/pyruvate $(\mathrm{S} / \mathrm{P})$ peaks were seen only in $3 / 4$ abscess (Table 3).

All spectroscopic parameters were non-significant in differentiation between primary and metastatic neoplastic lesions when the voxel measured inside the lesions; however, there is statistical significance for differentiation as regards the measured $\mathrm{Cho} / \mathrm{NAA}$ and $\mathrm{Ch} / \mathrm{Cr}$ ratios, $\left(P\right.$ value $\left.<0.001^{*}\right)$ (statically significant) when measured in the pre-lesional areas outside the tumor margin (Table 4). The overall MRS diagnosis of the all studied neoplastic and neoplastic lesions is approximately $95.3 \%$ sensitivity, $97.1 \%$ specificity, and $96 \%$ accuracy.

\section{DWI diagnosis}

In DWI, there was mixed diffusion restriction and facilitation in most of the studied lesions (68/100 lesions). The measured ADC values range showed overlap values in neoplastic and non-neoplastic lesions, it ranged between 0.6-1.7 with mean \pm SD approximate $0.88 \pm 0.31$ in neoplasms and between $0.37-3.9$ with mean \pm SD approximate $0.91 \pm 0.61$ in non-neoplastic lesions with no statistical significance for differentiation between neoplastic and non-neoplastic lesions, $P$ value $=0.236^{*}$ (Table 5).

In this work, the measured $\mathrm{ADC}$ values $(\mathrm{M} \pm \mathrm{SD})$ within the primary and metastatic neoplastic lesions were $0.75 \pm 0.16$ and $0.89 \pm 0.23$ respectively, with no significant difference $(P$ value $=0.165$ "insignificant"). Also, ADC value measurements outside the margin of primary and metastatic neoplastic lesions $(1.56 \pm 0.61$ and $1.71 \pm 0.25)$ showed no significant difference with insignificant $P$ value $=0.142$ (Table 6). The DWI with calculated ADC value results diagnosed the studied lesions with ratios approximate $87.6 \%$ sensitivity, $85.7 \%$ specificity, and $87 \%$ accuracy.

\section{Combined MRS and DWI with calculated ADC value results}

The combined MRS and DWI with calculated ADC value results when measured within the lesion and at the pre-lesional areas of the studied cerebral focal lesions succeeded in diagnosing 96 lesions out of the 100 studied lesions. However, there was no significant difference noted as regards the specificity, sensitivity, and accuracy ratios of combined DWI and MRS results when compared to MRS results alone (Table 7, Figs. 1, 2, 3, 4, and 5).

\section{Discussion}

A definite diagnosis and characterization of intra-cranial masses based on structural MRI alone may be difficult and MRS with other non-invasive techniques can represent an advance in the specificity of cerebral lesion diagnosis [16]. MRS limits the use of invasive diagnostic approaches such as brain biopsy [17].

This study revealed that conventional MRI with contrast-enhanced images could have low specificity in differentiation between primary or metastatic multiple cerebral neoplasms, and there was diagnostic overlap between some multiple neoplastic and non-neoplastic lesions with $89.33 \%$ sensitivity, $44 \%$ specificity, and $78 \%$ accuracy. This was in agreements with Omuro et al. who stated that differentiation between tumors and nonneoplastic lesions using conventional MRI may be challenging, while MRI is a sensitive technique for detection of brain lesions, the specificity and capability of it for distinguishing between benign and malignant lesions are limited, and with Karen et al. who said that although conventional MRI provides images with excellent structural

Table 6 DWI and ADC values within and outside tumor margins of the primary and metastatic neoplastic lesions

\begin{tabular}{llll}
\hline ADC value & Primary neoplastic & Metastatic neoplastic & $P$ value \\
\hline Range within $(M \pm S D)$ & $0.47-1,0.75 \pm 0.16$ & $\mathbf{0 . 6 1 - 1 . 2 , 0 . 8 9 \pm 0 . 2 3}$ & 0.165 insignificant \\
Range outside $(M \pm S D)$ & $0.91-2.3,1.56 \pm 0.61$ & $\mathbf{0 . 8 4 - 2 . 6 , 1 . 7 1 \pm 0 . 2 5}$ & $\mathbf{0 . 1 4 2}$ insignificant \\
\hline
\end{tabular}


Table 7 Sensitivity, specificity, and accuracy of conventional MRI, MRS, and DWI with ADC values for all studied lesions

\begin{tabular}{lllll}
\hline Modality & Sensitivity & Specificity & Accuracy & $P$ value \\
\hline Conventional MRI & $89.33 \%$ & $44.4 \%$ & $78 \%$ & Insignificant \\
MRS & $95.3 \%$ & $97.1 \%$ & $96 \%$ & Significant \\
$\begin{array}{l}\text { DWI + ADC } \\
\text { measurements }\end{array}$ & $87.6 \%$ & $85.7 \%$ & $87 \%$ & Insignificant \\
$\begin{array}{l}\text { Combined MRS + DWI } \\
\text { results }\end{array}$ & $95 \%$ & $97 \%$ & $96 \%$ & Significant \\
\hline
\end{tabular}

detail, it cannot always be used alone to accurately identify specific tumor type and grade or to differentiate neoplastic from non-neoplastic lesions [18, 19]. Sensitivity, specificity, PPV, and NPV for determining a high-grade glioma with conventional MR imaging were $72.5 \%, 65.0 \%, 86.1 \%$, and $44.1 \%$, respectively [20].

MRS can add clinically relevant information about metabolites in common brain abnormalities. It is clinically ready for diagnostic, prognostic, and treatment assessment of brain tumors; various demyelinating disorders, and infectious brain lesions [21]. Cho/Cr, Cho/NAA,
$\mathrm{NAA} / \mathrm{Cho}$, and NAA/Cr ratios are the most concerned ratios. This may be of help in the diagnosis and differentiation between many lesions that may be confusing on the conventional MRI imaging [22].

According to our MRS results, the studied 100 lesions were classified into non-neoplastic and neoplastic lesions, and there was statistical significance for their differentiation by MRS as regards the Cho/Cr, Cho/NAA, $\mathrm{NAA} / \mathrm{Cr}$, Cho/NAA + $\mathrm{Cr}$, and NAA/Cho ratios $(\mathrm{M} \pm$ SD) with $P<0.001$ (significant). This was coincidence with many other studies that revealed large differences in metabolite levels in acute stroke, chronic multiple sclerosis, and brain tumors [23-25] and Brandáo et al. study which concluded that increase in Cho levels and Cho/Cr and Cho/NAA ratios is highly suggestive of neoplasm [26]. There is extensive literature demonstrating the metabolite ratios of $\mathrm{Cho} / \mathrm{Cr}, \mathrm{NAA} / \mathrm{Cr}$, as well as the presence of lipids and lactate to be useful in grading tumors and predicting tumor malignancy [20]. While Moller-hartman et al. stated that the abnormality specific to tumors was the elevated $\mathrm{CHO}$ [27]. Also, our results showed that most of the neoplastic and non-

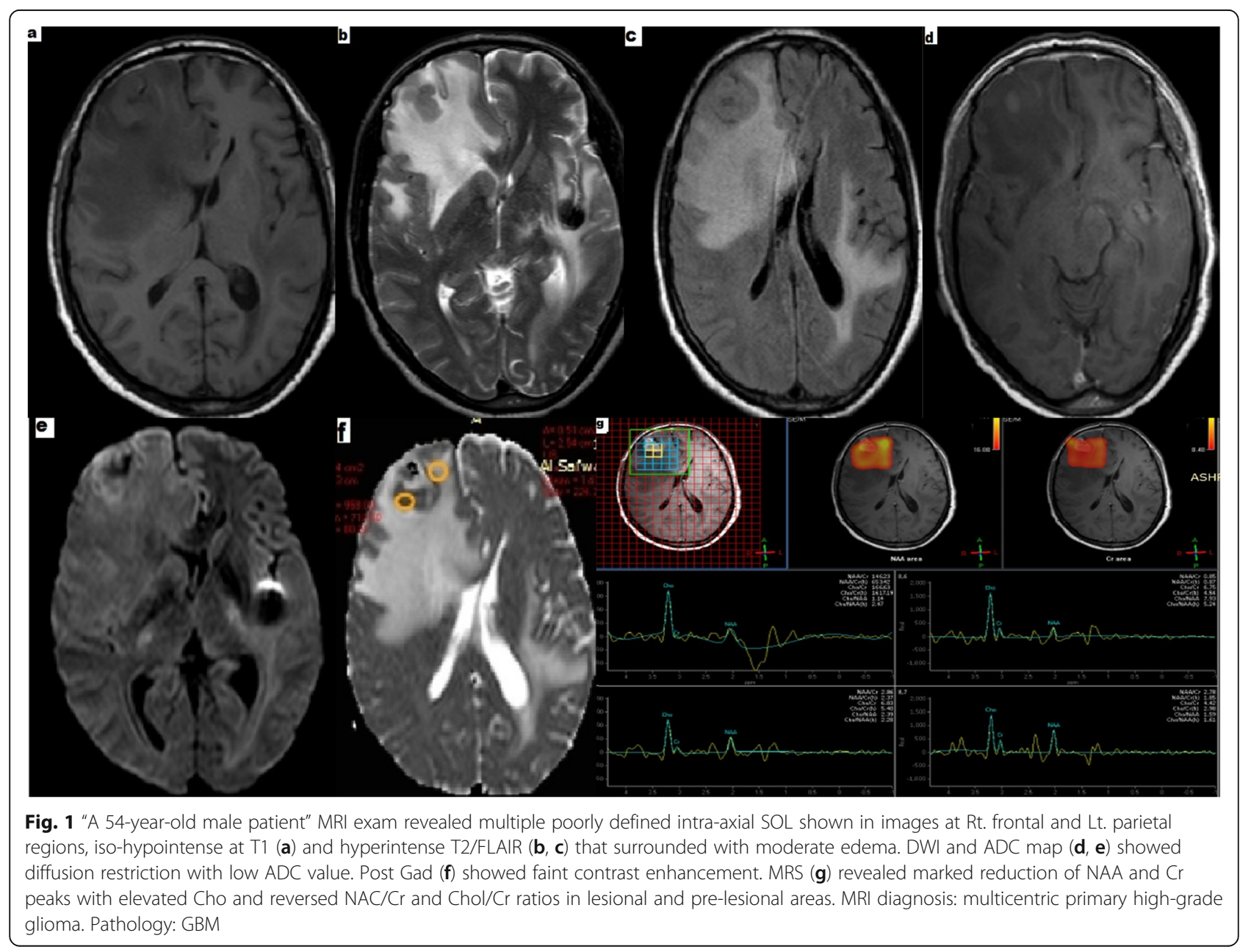




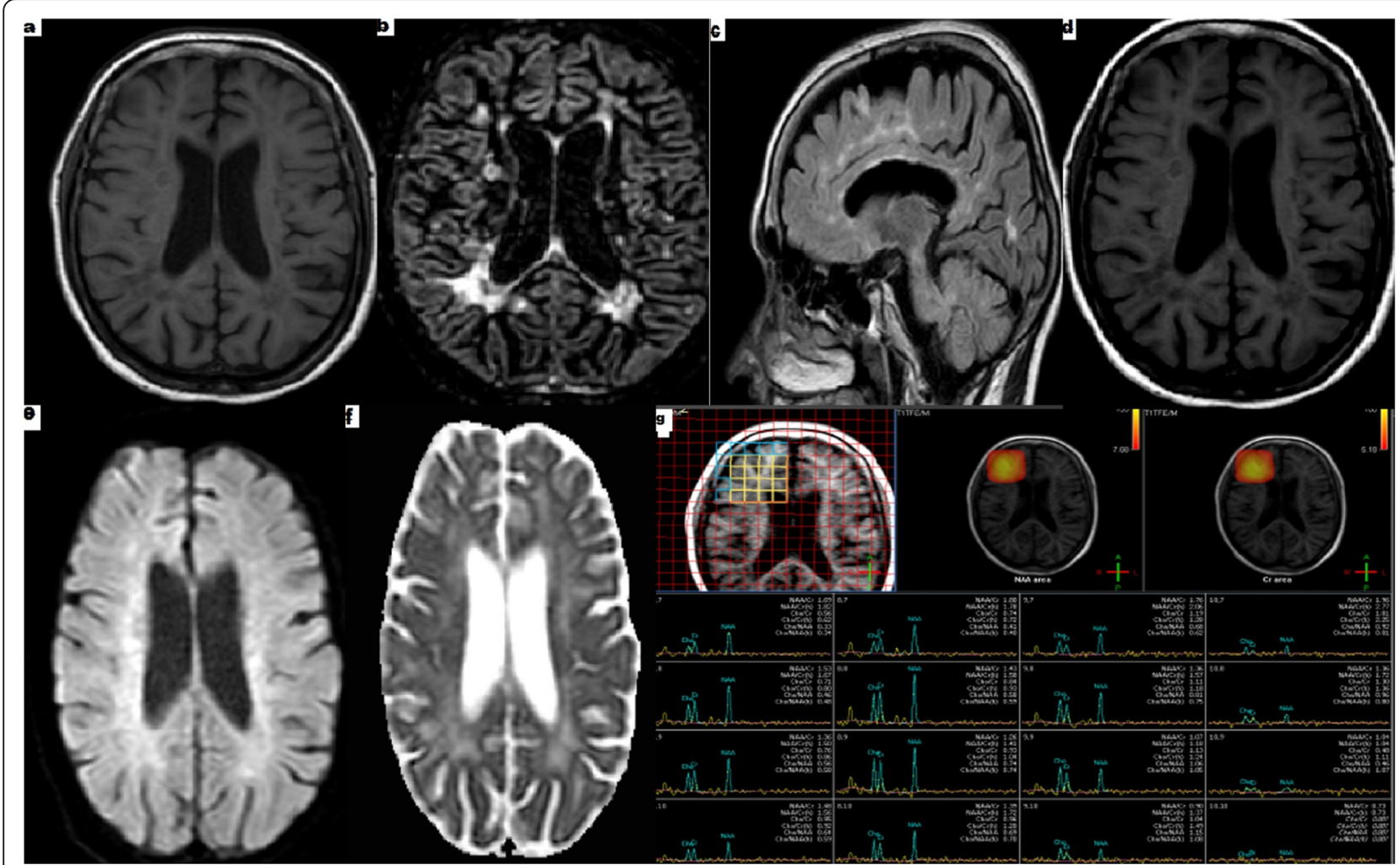

Fig. 2 "A 33-year-old female patient" MRI exam revealed multiple well-defined abnormal signal intensity foci scattered at the white matter of both cerebral hemispheres and posterior fossa that exhibit iso-hypointense signal at T1 (a) and hyperintense at 3D Gr and FLAIR (b, c) with no associated edema. DWI and ADC map (d, e) showed mixed diffusion changes (restriction/T2 shin effect). Post Gad (f) showed no contrast enhancement. MRS (g) revealed no significant metabolic changes with maintained ratios at the lesional and pre-lesional areas. MRI diagnosis: multiple sclerosis. Clinical diagnosis and follow-up: MS

neoplastic lesions showed elevated lipid and lactate peak respectively which increased with tumor aggressiveness. This agreed with Nooman et al. [28] results that revealed lactate and lipid levels in low and high grade tumors but with no significant difference between primary and metastatic tumors and Van-der $G$ who stated that the presence of the lactate peaks was usually consistent with aggressive tumors [29].

Other metabolites such as AA, A, and S/P peaks were seen only in $3 / 4$ abscess. This agree with the results that summarized spectral characteristics of intracranial abscesses as presence of cytosolic amino acids (detected at $0.9 \mathrm{ppm}$ ): presence of lactate (detected at $1.3 \mathrm{ppm}$ ), acetate (detected at $1.92 \mathrm{ppm}$ ), succinate (detected at 2.4 ppm), and occasionally lipids (detected at 0.8-1.2 ppm) [30, 31].

In our studies, all spectroscopic parameters showed non-significant results for differentiation between primary and metastatic neoplastic lesions when measured within the lesions; however, there was a statistical significance of Cho/NAA and $\mathrm{Ch} / \mathrm{Cr}$ ratios $\left(P\right.$ value $\left.<0.001^{*}\right)$ for such differentiation when measured in the perilesional regions. This was in agreements with many other studies who found that investigation of peri-enhancing tumor regions may be useful for discriminating metastases from primary brain tumors, whereas gliomas are often invasive lesions with elevated Cho in surrounding tissue, metastatic lesions tend to be more encapsulated and do not show high Cho signals or other abnormalities outside the region of enhancement [32-34].

The results of this work revealed that there were mixed diffusion changes in most of the studied lesions and the measured ADC value ranges showed overlap in neoplastic and non-neoplastic lesions with no statistical significance for their differentiation. Also, the calculated ADC values showed no significant difference either measured within or outside the margins of primary and metastatic neoplasms, $P$ value $=0.165$ and 0.142 respectively "insignificant."

This was matched with results of Fawzy et al, who said that regarding DWI, we found a heterogeneous signal and different values for ADC according to the lesions' consistency and there was no significant correlation between the histological components and the ADC values [35]. Also, Al-Okaili et al. and other studies found that 


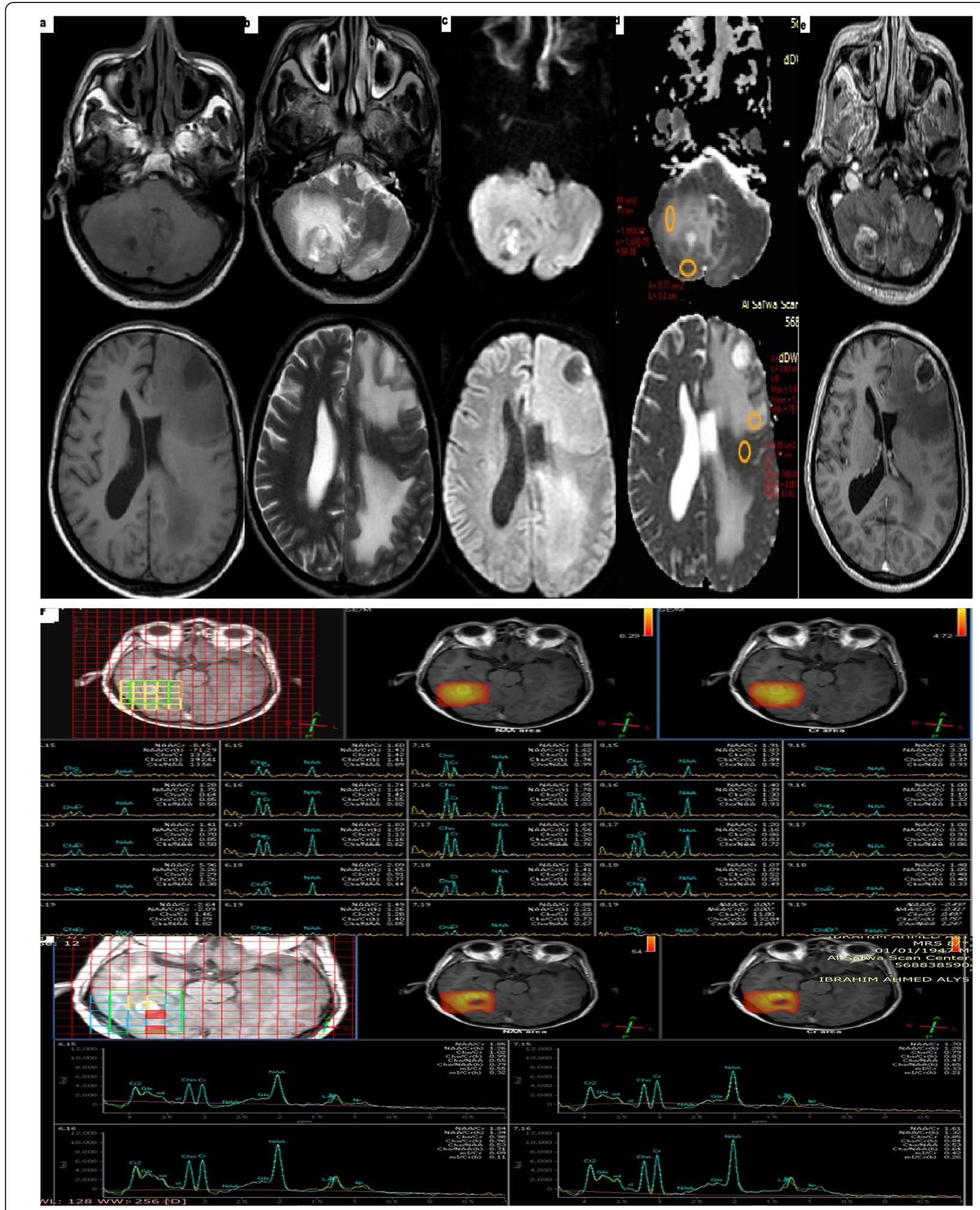

Fig. 3 "A 52-year-old female patient" with known primary breast Ca, MRI exam revealed multiple semi-defined intra-axial SOLs shown in images at cerebellum and both cerebral hemispheres, iso-hypointense at T1 (a) and hyper at T2 (b) that surrounded with moderate edema. DWI and ADC map (c, d) showed diffusion restriction with low ADC values. Post Gad (e) showed peripheral nodular contrast enhancement. MRS (f, $\mathbf{g}$ ) revealed marked reduction of NAA and $\mathrm{Cr}$ peaks with elevated Cho within the lesions with normal pre-lesional metabolites. MRI diagnosis: metastatic breast Ca. Pathology: proved metastatic deposits 

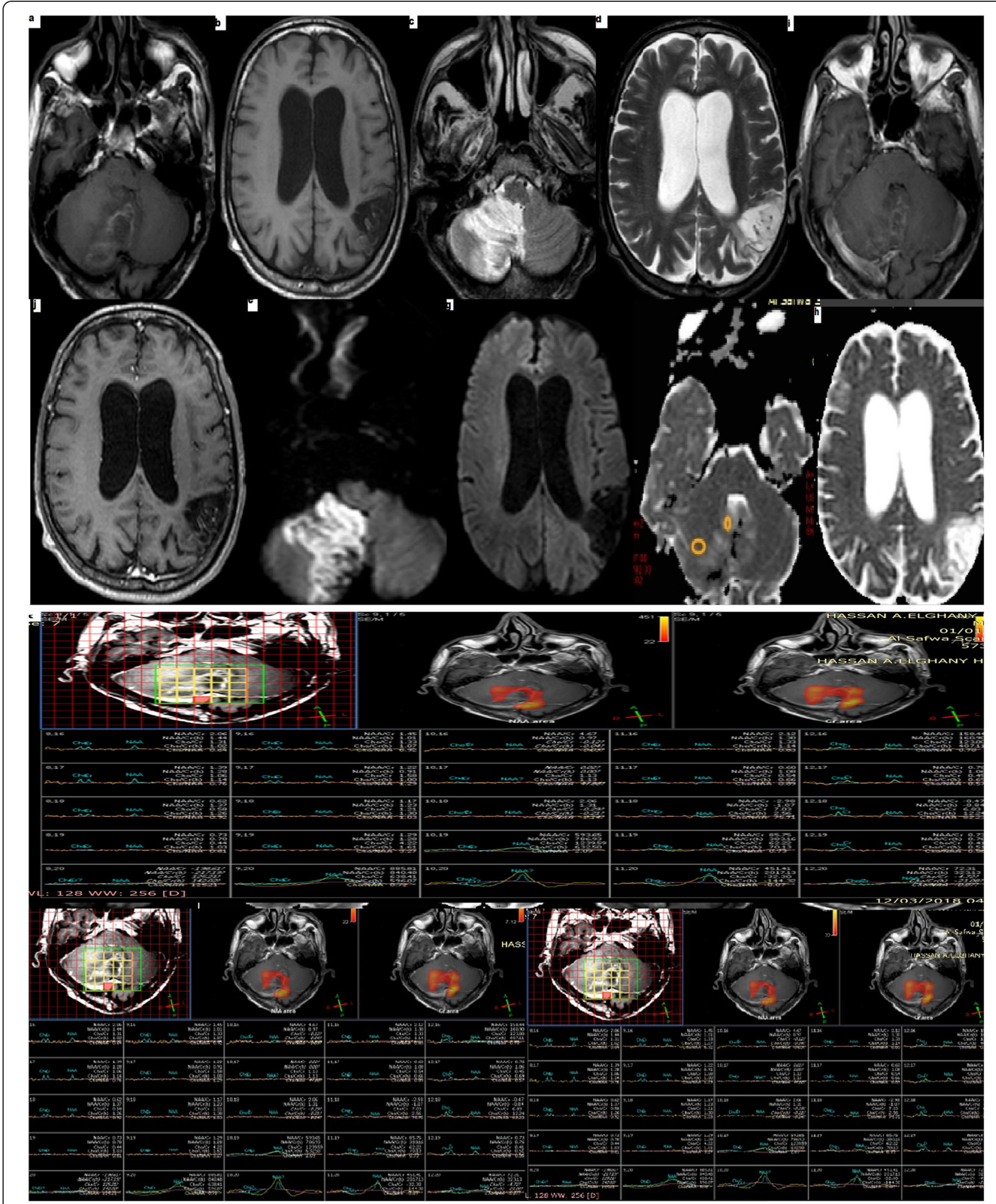

Fig. 4 "A 42-year-old female patient" MRI exam revealed multiple well-defined abnormal signal intensity lesions at the cerebellum and Lt. occipital regions that exhibit hypointense signal at T1 with hyperintense foci within cerebellum lesion (suggesting blood) (a, b) and hyperintense signals at T2 (c, d) with no associated edema. DWI and ADC map (e, $\mathbf{f}$ and $\mathbf{g}, \mathbf{h})$ showed mixed diffusion changes (restriction $/ T 2$ shin effect at cerebellum and facilitated diffusion of occipital lesion). Post Gad (i, j) showed no contrast appreciable enhancement. MRS (k) revealed no significant changes either of NAA, $\mathrm{Cr}$, or Cho peaks with maintained ratios at the lesional and pre-lesional areas. MRI diagnosis: multiple infarcts of different chronologic ages that proved by clinical diagnosis and follow-up 


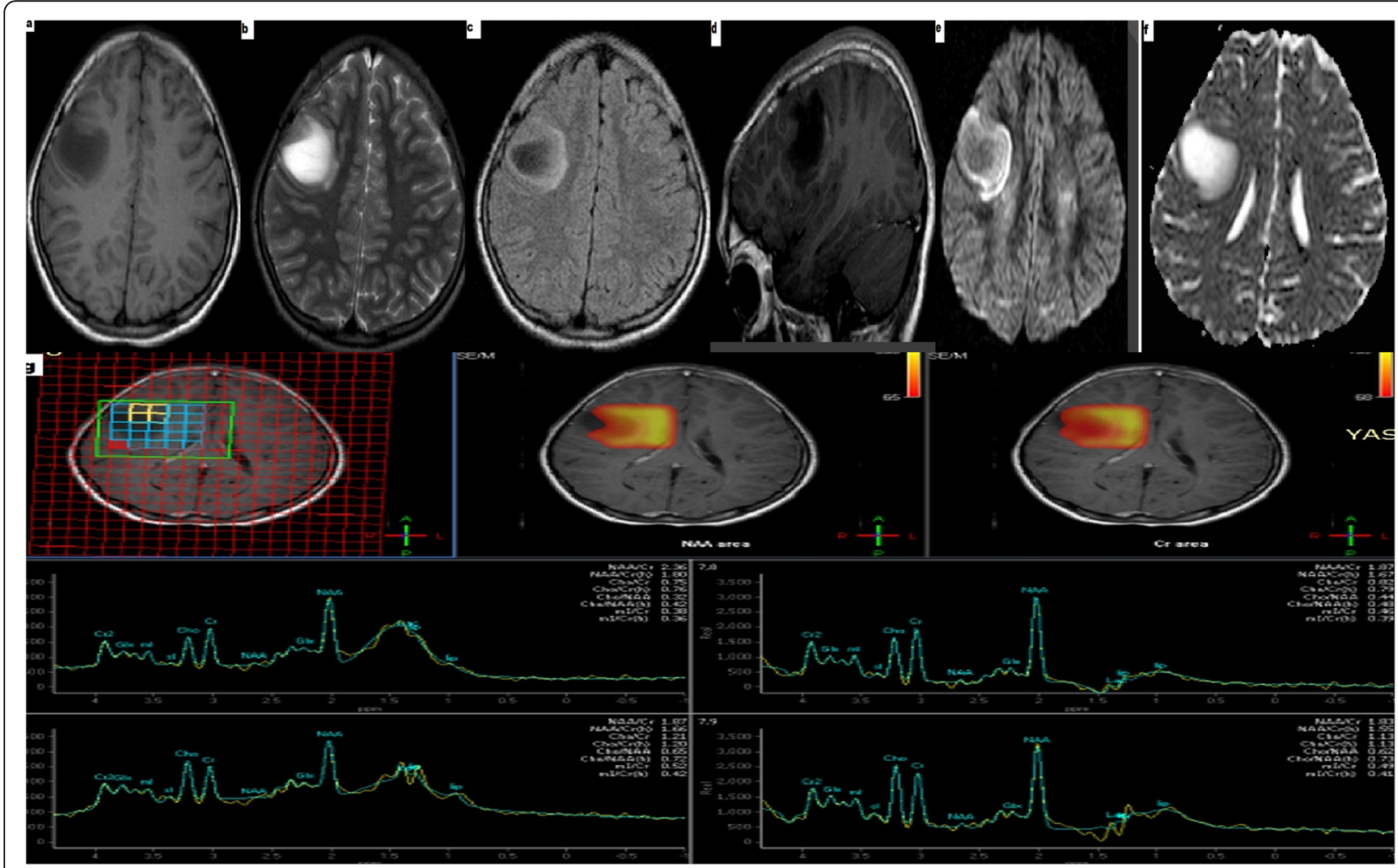

Fig. 5 "An 11-year-old female patient" MRI exam revealed a poorly defined lesions at the Lt. high parietal region that exhibit hypointense signal at T1 (a) and hyperintense signals with surrounding hypointense rim at T2 and FLAIR (b, c) and minimal perifocal edema. DWI and ADC map (d, e) showed mixed diffusion changes (restriction/T2 shin effect) with high ADC value. Post Gad (f) showed marginal ring contrast enhancement. MRS $(\mathbf{g}, \mathbf{h})$ revealed no significant changes either of NAA, Cr, or Cho peaks with presence of AA "glycine (Gly) at $3.56 \mathrm{ppm}$; succinate (S) at $2.4 \mathrm{ppm}$; lipid/lactate (Lip/L) at $1.3 \mathrm{ppm}$ at short MRS. Also, there is a well-defined abnormal signal intensity area seen at the Rt. frontal region (not included in selected images). MRI diagnosis: inflammatory process with Rt. parietal pyogenic brain abscess that proved on surgical aspiration of pus contents

DWI feature for primary neoplasms is variable and although the ADC value of high-grade gliomas has been shown to be lower than that of low grade gliomas, there is substantial overlap; thus, ADC maps alone are insufficient for predicting type and grade of glial neoplasms and it could not differentiate between these tumors [36-39].

Our works showed that the combined MRS and DWI with calculated ADC values added to cMRI results succeeded in diagnosing $96 \%$ of the studied lesions. This was in agreement with Abdel-Monem et al. study which concluded that the combination of MRS with cMRI and calculated ADC values added more information in the differentiation of brain tumors and were more useful when done together than each alone which markedly elevate the diagnostic accuracy rate [40].

There were a few limitations of this study. First, the low number of some studied lesions, especially that of non-neoplastic etiology such as tuberculoma, abscesses, and vascular lesions. Second, when we applied DWI, there was difficulty in positioning the region of interest in cystic/necrotic lesions as well as in the pre-lesional regions of some lesions that are not associated with edema. Lastly, the diagnosis of multicentric cerebral focal lesions with primary and secondary brain neoplasm differentiation is still a challenging problem based on conventional MRI alone, and according to the results of this study, it was found that a combination of proton MRS and DWI with ADC calculation can provide additive information helping in lesion characterization which improves the diagnosis and management plane.

\section{Conclusion}

MRS was found to be a non-invasive and accurate diagnostic tool that is superior to DW-MRI with ADC value measurements in the differentiation and therapeutic planning of multicentric cerebral focal lesions. 


\section{Abbreviations}

Cho: Choline; Cr: Creatine; DWI-MRI: Diffusion weighted magnetic resonance imaging; MCFL: Multicentric cerebral focal lesions; MRS: Magnetic resonance spectroscopy; NAA: N-acetyl aspartate; NPV: Negative predictive value; PPV: Positive predictive value

\section{Acknowledgements}

Not applicable, as all participants is co-author for this research.

\section{Authors' contributions}

HAK designed the work and helped in the data collection, images reviewing, interpretation, and writing of the manuscript. HM shared in writing of the manuscript and edition. All authors have read and approved the final manuscript.

\section{Funding}

Not applicable

\section{Availability of data and materials}

The dataset analyses during the current study are available from the corresponding author on reasonable request. All is available with the authors upon request.

\section{Ethics approval and consent to participate}

This study was done after the approval from AL-Azhar University HospitalFaculty of Medicine-Assuit and after patient agree verbal consent (as the patients not exposed to any type of surgical or intervention maneuver). This study was done during the period from January 2018 to August 2018. Approval number 7-1- 2018 "Paper code-Meeting code-Year"

\section{Consent for publication}

All patients included in this research gave written informed consent to publish the data contained within this study. If the patient was less than 16 years old, deceased, or unconscious when consent for publication was requested, written informed consent for the publication of this data was given by their parent or legal guardian.

\section{Competing interests}

The authors declare that they have no competing interests.

\section{Author details}

${ }^{1}$ Radio-diagnosis Department, Faculty of Medicine, Al-Azhar University, Assuit, Egypt. ${ }^{2}$ Radio-therapy Department, Faculty of Medicine, Minia University, El-Minia, Egypt.

\section{Received: 13 January 2020 Accepted: 20 February 2020}

Published online: 24 April 2020

\section{References}

1. Clare HC, Ingeborg F, David M et al (2009) Intracranial lesions mimicking neoplasms. Arch Pathol Lab Med 133(1):101-123

2. Hermann EJ, Hattingen E, Krauss JK et al (2008) Stereotactic biopsy in gliomas guided by 3-tesla $1 \mathrm{H}$-chemical-shift imaging of choline. Stereotact Funct Neurosurg 86(5):300-307

3. Di Costanzo A, Scarabino T, Trojsi F et al (2006) Multiparametric 3T MR approach to the assessment of cerebral gliomas: tumor extent and malignancy. Neuroradiology 48(9):622-631

4. Yu X, Liu Z, Tian Z, Li S et al (2000) Stereotactic biopsy for intracranial space-occupying lesions: clinical analysis of 550 cases. Stereotact Funct Neurosurg 75:103-108

5. Delorme S, Weber M-A (2006) Applications of MRS in the evaluation of focal malignant brain lesions. Cancer Imaging 6:95-99

6. Butzen J, Prost R, Chetty V et al (2000) Discrimination between neoplastic and non-neoplastic brain lesions by use of proton MR spectroscopy: the limits of accuracy with a logistic regression model. AJNR 21:1213-1219

7. Stadlbauer A, Gruber S, Nimsky C et al (2006) Pre-operative grading of gliomas by using metabolite quantification with high-spatial-resolution proton MR spectroscopic imaging. Radiology 238:958-969

8. Razek AA, Abdalla A, Gaber NA et al (2013) Proton MR Spectroscopy of the brain in children with neuronopathic Gaucher's disease. Eur Radiol 23:3005-3011
9. Razek AA, Abdalla A, Ezzat A et al (2014) Minimal hepatic encephalopathy in children with liver cirrhosis: diffusion-weighted MR imaging and proton MR spectroscopy of the brain. Neuroradiology 56:885-891

10. Crawford FW, Khayal IS, McGue C et al (2009) Relationship of presurgery metabolic and physiological MR imaging parameters to survival for patients with untreated GBM. J Neurooncol 91(3):337-351

11. Sepahdari AR, Politi LS, Aakalu VK et al (2014) Diffusion-weighted imaging of orbital masses: multi-institutional data support a 2-ADC threshold model to categorize lesions as benign, malignant, or indeterminate. AJNR Am J Neuroradiol 35:170-175

12. Abdel Razek AA, Gaballa G, Denewer A, Tawakol I (2010) Diffusion weighted MR imaging of the breast. Acad Radiol 17:382-386

13. Razek AA (2012) Diffusion magnetic resonance imaging of chest tumors Cancer Imaging 12:452-463

14. Schaefer PW, Grant PE, Gonzalez RG (2000) Diffusion-weighted MR imaging of the brain. Radiology 217:331-345

15. Bulakbasi N, Kocaoglu M, Ors F et al (2003) Combination of singlevoxel proton MR spectroscopy and apparent diffusion coefficient calculation in the evaluation of common brain tumors. Am J Neuroradiol 24:225-233

16. Aasly J, Silfvenius $H$, Aas TC et al (1999) Proton magnetic resonance spectroscopy of brain biopsies from patients with intractable epilepsy. Epilepsy Res 35:211-217

17. Burger PC, Scheithauer BW, Paulus W et al (2000) Pathology and genetics of tumours of the nervous system. International Agency for Research on Cancer, Lyon, pp 45-51

18. Omuro AM, Leite CC, Mokhtari K et al (2006) Pitfalls in the diagnosis of brain tumours. Lancet Neurol 5(11):937-948

19. Karen M, Simrandip KG, Niloufar Z et al (2018) Evaluation of the added value of $1 \mathrm{H}$-magnetic resonance spectroscopy for the diagnosis of pediatric brain lesions in clinical practice. Neuro-Oncology Practice 5(1):18-27

20. Meng L, Stanley Y, Hao W et al (2003) Glioma Grading: Sensitivity, Specificity, and Predictive Values of Perfusion MR Imaging and Proton MR Spectroscopic Imaging Compared with Conventional MR Imaging. Am J Neuroradiolo 24(10):1989-1998

21. Gülin O, Jeffry RA, Peter BB et al (2014) Clinical Proton MR Spectroscopy in Central Nervous System Disorders. Radiology 270(3):658-679

22. PB B (2005) In: WA GJH, Parker PB (eds) Clinical MR Neuroimaging: diffusion, perfusion, and spectroscopy. Cambridge University Press, Capetown

23. Bruhn H, Frahm J, Gyngell ML et al (1989) Cerebral metabolism in man after acute stroke: new observations using localized proton NMR spectroscopy. Magn Reson Med 9(1):126-131

24. Arnold DL, Matthews PM, Francis G et al (1990) Proton magnetic resonance spectroscopy of human brain in vivo in the evaluation of multiple sclerosis: assessment of the load of disease. Magn Reson Med 14(1):154-159

25. Bruhn H, Frahm J, Gyngell ML et al (1989) Noninvasive differentiation of tumors with use of localized H-1 MR spectroscopy in vivo: initial experience in patients with cerebral tumors. Radiology 172(2):541-548

26. Brandáo LA, Domingues RC (2004) MR spectroscopy of the brain. Philadelphia:Lippincott Williams \& Wilkins 5:33-85

27. Möller-Hartmann W, Herminghaus S, Krings T et al (2002) Clinical application of proton magnetic resonance spectroscopy in the diagnosis of intra-cranial lesions. Neuroradilogy 44:371-381

28. Nooman AD, Mohamed NA, Mohamed AR et al (2016) Role of magnetic resonance spectroscopy \&diffusion weighted imaging in differentiation of supratentorial brain tumors. The Egyptian J Radiol Nucl Med 47:1037-1042

29. Van Der Graaf M (2010) In vivo magnetic resonance spectroscopy: basic methodology and clinical applications. Eur Biophys J 39(4):527-540

30. Lai PH, Ho JT, Chen WL et al (2002) Brain abscess and necrotic brain tumor: discrimination with proton MR spectroscopy and diffusionweighted imaging. AJNR Am J Neuroradiol 23(8):1369-1377

31. Akutsu $H$, Matsumura A, Isobe T et al (2002) Chronological change of brain abscess in (1)H magnetic resonance spectroscopy. Neuroradiology 44(7):574-578

32. Fan G, Sun B, Wu Z et al (2004) In vivo single-voxel proton MR spectroscopy in the differentiation of high-grade gliomas and solitary metastases. Clin Radiol 59(1):77-85

33. Chiang IC, Kuo YT, Lu CY et al (2004) Distinction between highgrade gliomas and solitary metastases using peritumoral 3-T magnetic resonance spectroscopy, diffusion, and perfusion imagings. Neuroradiology 46(8):619-627

34. Ishimaru H, Morikawa M, Iwanaga S et al (2001) Differentiation between high-grade glioma and metastatic brain tumor using single-voxel proton MR spectroscopy. Eur Radiol 11(9):1784-1791 
35. Fawzy FM, Ibrahim DH, Gouda MA (2014) MR spectroscopy and diffusion MR imaging in characterization of common sellar and suprasellar neoplastic lesions. Egypt J Radiol Nucl Med 45:859-867

36. Al-Okaili RN, Krejza J, Wang S et al (2006) Advanced MR Imaging Techniques in the Diagnosis of Intra-axial Brain Tumors in Adults. RadioGraphics 26:S173-S189

37. Pavlisa G, Rodos M, Gordana P (2009) The differences of water diffusion between brain tissue infiltrated by tumor and peritumoral vasogenic odeama. Clin Imag 33:96-101

38. Ja-Lee E, TerBrugge K, Choil DS (2011) Diagnostic value of peritumoral minimum apparent diffusion coefficient for differentiation between glioblastoma multiforme from solitary metastatic lesions. Am J Radiol 196: 71-76

39. Ohba S, Ushioda T, Nakagawa T (2011) Diffusion magnetic resonance imaging for enhanced visualization of malignant cerebral tumors and abscesses. Neurol India 59:674-678

40. Abdel-Monem N, Darweesh ME, Badawy MH et al (2014) Magnetic resonance spectroscopy and diffusion imaging in the evaluation of neoplastic brain lesions. Egypt j radiol nucl med 45:485-493

\section{Publisher's Note}

Springer Nature remains neutral with regard to jurisdictional claims in published maps and institutional affiliations.

\section{Submit your manuscript to a SpringerOpen ${ }^{\odot}$ journal and benefit from:}

- Convenient online submission

- Rigorous peer review

- Open access: articles freely available online

High visibility within the field

- Retaining the copyright to your article

Submit your next manuscript at $\boldsymbol{\nabla}$ springeropen.com 